\title{
Enhancement of the IceCube surface instrumentation by a hybrid radio and scintillation detector array
}

\section{S. Shefali, The IceCube Collaboration}

E-mail: shefali.shefali@kit.edu

The IceCube Neutrino Observatory is a cubic kilometer scale detector deployed in the antarctic ice, capable of detecting neutrinos of energies ranging from approximately $10 \mathrm{GeV}$ to $\mathrm{PeV}$ and above. In addition to being a powerful neutrino observatory, IceCube is extensively involved in cosmic ray physics. The surface array of IceCube, IceTop, consisting of frozen water tanks equipped with photomultipliers, detects secondary particles like electrons, protons and muons from cosmic ray air showers of energies up to $1 \mathrm{EeV}$. In addition, it is also used to function as a veto for the astrophysical neutrino searches and calibration detector for the IceCube in-ice instrumentation. Despite the valuable scientific results obtained so far, the snow accumulation on top of these detectors contributes to an increased energy uncertainty in the detected signals, and consequently, the shower reconstruction. Moreover, improvements to the array are needed to understand the astrophysics of the high-energy cosmic-ray sky. Enhancing IceTop with a hybrid array of scintillation detectors and radio antennas will lower the energy threshold for air-shower measurements, provide more efficient veto capabilities, enable the separation of the electromagnetic and muonic shower components and improve the detector calibration by compensating for snow accumulation. Following the success of the first prototype station consisting of three radio antennas and eight scintillation detectors deployed at the South Pole in 2018, the production of detectors for a total 32 stations is ongoing. The deployment status, calibration methods, and science goals of the enhancement will be discussed in this contribution.

Corresponding authors: S. Shefali ${ }^{1, *}$

1 Karlsruhe Institute of Technology, Institute for Astroparticle Physics, 76021 Karlsruhe, Germany

* Presenter

\footnotetext{
*** The European Physical Society Conference on High Energy Physics (EPS-HEP2021), ***

*** 26-30 July $2021 * * *$

*** Online conference, jointly organized by Universität Hamburg and the research center DESY ***
} 


\section{Introduction}

The surface array of IceCube, IceTop [1], consists of an array of 162 ice-Cherenkov detector tanks, placed on the surface of IceCube. IceTop is functioning as a cosmic-ray detector as well as a veto and calibration system for the IceCube in-ice neutrino detector. The yearly increasing snow accumulation on these surface detectors, however, results in an uncertainty in the detected signals, and consequently the shower reconstruction. An enhancement of the IceTop surface array is proposed to investigate this impact and improve the cosmic-ray measurements. This enhancement will consist of a total of 32 stations, each of which have 8 scintillation detectors and 3 radio antennascontrolled by a central DAQ system. The entire array is expected to be deployed by the Antarctic summer of 2026, and the footprint can be seen in Fig. 1.

In 2018, two prototype stations with different data acquisition systems were deployed at the South
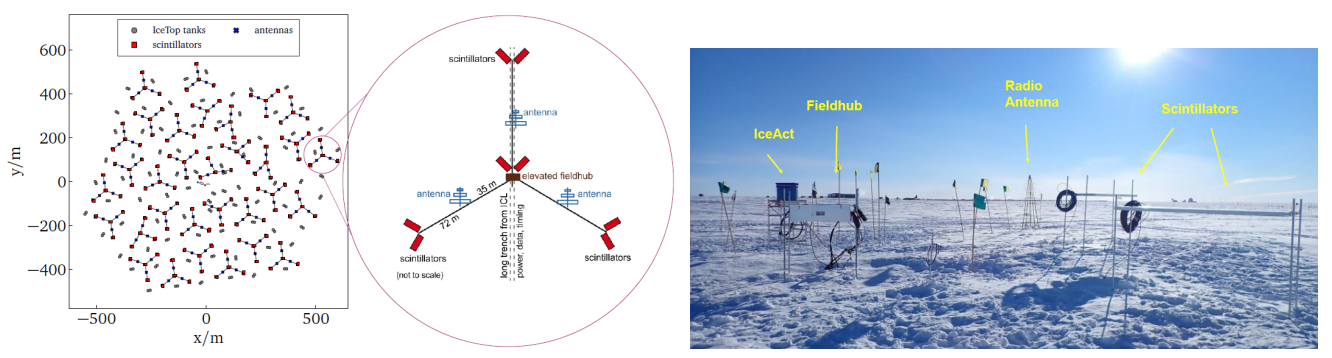

Figure 1: Left: Layout of the 32 station array for the surface array enhancement (Middle: A closer look at the individual stations, with the planned orientation of the detectors and DAQ system [2]). Right: The prototype station deployed at the Pole in January 2020.

Pole. The results for both the stations were observed to agree very well with the requirements as well as each other [3]. Subsequently, an upgraded prototype station was set up in January 2020 (Fig. 1). The station has been fully operational after an initial commissioning period, and we have been able to obtain the first coincident measurements of cosmic ray air showers using a combination of the scintillation detectors, radio antennas and IceTop.

The surface array enhancement has numerous scientific goals to further the study of cosmic rays. Along with providing an independent detection method to better understand hadronic interactions and yield an improved air-shower reconstruction, the surface array enhancement will also significantly improve the veto capabilities of the existing IceTop array. The addition of these scintillation detectors will lower the energy threshold by doubling the sensitive detection area at the same exposure. Finally, an important contribution of these detectors will be research and development for the proposed enhancement of IceCube, IceCube-Gen2 [4].

\section{Enhancement Detectors}

Similar to the prototype station, a single station for the enhancement is designed to have 8 scintillation detectors, 3 radio antennas and a central data acquisition system (DAQ) which is an adapted version of the Transportable Array for eXtremely large area Instrumentation studies (TAXI) [5]. The TAXI board has an embedded Linux operating system running on a microprocessor (Stamp9G45 by TasKit $\mathrm{GmbH}$ ), 3 DRS4 samplers for converting analog signals from the radio antennas and an FPGA (SPARTAN-6 by XILINX) for processing the signals [5]. Nanosecond precision timing is 


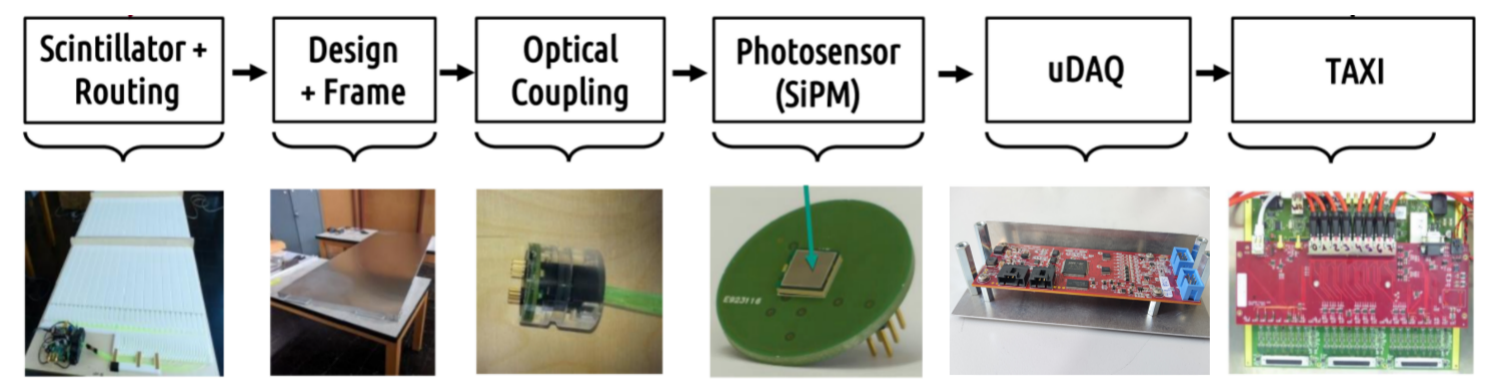

Figure 2: The main components of scintillation detectors: The scintillation bars along with optical fibre routings are coupled to the SiPM which is read out using the microDAQ board. The microDAQ is in turn connected to central DAQ system, the TAXI.

provided to the DAQ system by the White Rabbit switch and node combination [6]. The TAXI is responsible for providing power, communication and timing for the scintillation detectors as well. The scintillation detectors (Fig. 2) [7] consist of 16 plastic scintillation bars made of polystyrene (produced at FNAL) and have a sensitive area of $1.5 \mathrm{~m}^{2}$ each, Wavelength-shifting fibers (Y-11(300) by Kuraray) are routed through two holes in the scintillation bars and glued to the Silicon Photomultiplier (SiPM) using a PMMA coupler. Since the SiPM sensitivity depends on the temperature, a temperature sensor is placed next to the SiPM. A custom microDAQ board is used to digitize and read out the SiPM signals, before transferring the processed data to the central DAQ system. The radio emission from the air showers is detected by dual polarisation Log-Periodic Dipole Array (LPDA) antennas [8]. Each polarisation of the antenna has an individual Low Noise Amplifier (LNA) located at the top of the antennas. These antennas are manufactured to operate in the range of $50 \mathrm{MHz}-350 \mathrm{MHz}$ for the surface array enhancement.

\section{Preliminary Results}

After the initial commissioning period in 2018, the prototype station deployed in 2020, has been recording air shower data and measured the first coincident events with the primary IceTop array.

When an ionizing particle crosses the scintillation detectors, the produced scintillation light is collected and guided via the wavelength-shifting fibers to the SiPM. Each event is recorded as the amount of charge deposited in units of ADC counts. The data are then histogrammed and used for gain calculations and MIP (Minimum Ionising Particle) peak localization. The MIP is obtained by fitting a Gaussian to the distribution, after removing the soft trigger baseline. An example of histogrammed data from one of the scintillation panels is shown in Fig. 3.

For the radio data, each trace is split into four and then sampled in parallel four times to suppress noise spikes. The median of the four traces is taken as the measured signal. The background spectrum is observed to agree quite well for all three antennas [9].

The access to air shower data from three independent sources, namely, scintillators, radio and IceCube (IceTop) poses a favorable opportunity to investigate coincident events. Since the radio antennas are triggered by scintillation detectors, their trigger time is used. For scintillators the timestamp of the first panel hit is taken into account. Finally, for IceTop data the reconstructed time of the shower hitting the surface is used. The events coincident within $2 \mu \mathrm{s}$ for all three sources, are considered a single coincident event. The shower direction can be reconstructed for these 

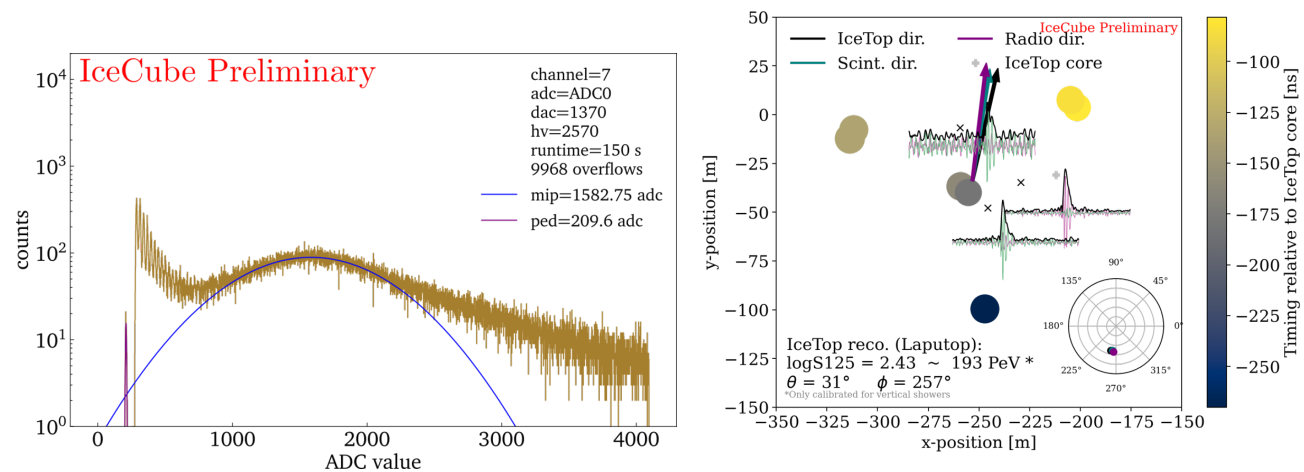

Figure 3: Left: Scintillation charge distribution for a 150s long measurement run as a histogram is shown. The MIP is fit with a Gaussian function. Right: An air shower event observed by all three detectors, namely, scintillators, radio and IceTop.

coincident events from each individual detector system [10]. An example of such an event observed by the prototype station is shown in Fig. 3, where the circles signify the scintillator locations and the ' $x$ ' marks the radio detectors. The size of the circles indicates the amount of charge recorded by the scintillators, and their colors represent the time of this charge deposit with respect to the IceTop. The ' + ' is the core position reconstructed by IceTop. Finally, the three arrows indicate a reconstructed direction of the shower for the three detectors.

In conclusion, the prototype station for the future surface array enhancement has been presented. Its operation under harsh conditions at the South pole, and successful air shower data retrieval has proven to be very promising for future deployment. The series production of detectors for the full 32-station array is underway. It is also planned to achieve an automated gain stabilization for a future seamlessly functional array.

\section{References}

[1] R. Abbasi et al, IceTop: The surface component of IceCube NIM A700:188,2013

[2] IceCube Collaboration, A. Leszczyńska, M.Plum, Simulation and Reconstruction Study of a Future Surface Scintillator Array at the IceCube Neutrino Observatory, PoS ICRC(2019)332

[3] IceCube Collaboration, T. Huber, J. Kelley, S. Kunwar, and D. Tosi The Scintillator Upgrade of IceTop: Performance of the prototype array, PoS ICRC(2019)309

[4] IceCube-Gen2 Collaboration, M. G. Artsen, IceCube-Gen2: the window to the extreme Universe, J.Phys.G 48 (2021) 6, 060501

[5] T. Karg, A. Haungs, M. Kleifges, R. Nahnhauer, and K. H. Sulanke, 6th International Workshop on Acoustic and Radio EeV Neutrino Detection Activities (ARENA 2014) Annapolis, MD (2014)

[6] P.P.M. Jansweijer, H.Z. Peek, E. de Wolf, White Rabbit: Sub-nanosecond timing over Ethernet, Nucl. Instrum. Meth. A 725 (2013) 187-190

[7] IceCube Collaboration, A. Haungs, A Scintillator and Radio Enhancement of the IceCube Surface Detector Array, EPJ Web Conf., 210, 2019 Ultra High Energy Cosmic Rays 2018 (UHECR 2018)

[8] E. de Lera Acedo, A. J. Faulkner and J. G. Bij de Vaate, SKA low frequency aperture array, USNC-URSI NRSM (2016)

[9] IceCube Collaboration, M. Oehler, R. Turcotte-Tardif, Development of a scintillation and radio hybrid detector array at the South Pole, PoS ICRC(2021)225

[10] IceCube Collaboration, H. Dujmovic, A. Coleman, M. Oehler, First air-shower measurements with the prototype station of the IceCube surface enhancement, PoS ICRC(2021)314 